\title{
Animal Models for the Study of Osteomyelitis
}

\author{
Mitul Patel, M.D., ${ }^{1}$ Yuri Rojavin, M.D., ${ }^{1}$ Amir A. Jamali, M.D., ${ }^{2}$ \\ Samantha J. Wasielewski, B.S., ${ }^{1}$ and Christopher J. Salgado, M.D. ${ }^{3}$
}

Osteomyelitis is an acute or chronic inflammatory process of the bone and its related structures secondary to an infection with pyogenic organisms. Because of the variety in disease presentations and pathophysiology of osteomyelitis, it is very difficult to evaluate in clinical studies. Therefore, animal models have been created for in vivo experimentation. A PubMed and OVID search was performed on March 31, 2008, using keywords osteomyelitis, animal model (rabbit, rat, mouse, avian, dog, sheep, and goat), and experimental osteomyelitis. The objective of this review was to provide a literature review of the animal models created to study osteomyelitis. The models were chosen based on historical relevance and clinical applicability. Numerous animal models exist to study both acute and chronic osteomyelitis. Many models have been created that allow investigators to study various aspects in the treatment and diagnosis of osteomyelitis. Based on the needs of investigators, an animal model must be carefully selected for ideal research, as no single model encompasses all aspects of osteomyelitis.

KEYWORDS: Osteomyelitis, animal models, rabbit, goat, mouse, canine

$\mathrm{O}_{\text {steomyelitis is an acute or chronic inflamma- }}$ tory process of the bone and its related structures secondary to an infection with pyogenic organisms. ${ }^{1}$ Osteomyelitis was classified by Waldvogel et al into three groups: hematogenous osteomyelitis, contiguous osteomyelitis, and osteomyelitis associated with peripheral vascular disease. ${ }^{2}$ Clinically, osteomyelitis was divided into 12 categories by Cierny et al to help determine appropriate management. ${ }^{3}$

Along with the variety of presentations of osteomyelitis, several confounding variables, such as a low incidence rate of osteomyelitis, a variety of pathogenic organisms, antibiotic sensitivity, and anatomic locations of the disease, prevent these classifications from being easily controlled in clinical studies. ${ }^{4}$ Secondary to the difficulty in creating a controlled clinical model to study

${ }^{1}$ Department of Surgery, Cooper University Hospital, Robert Wood Johnson Medical School, Camden, New Jersey; ${ }^{2}$ Department of Orthopedic Surgery, UC Davis Medical Center, Sacramento, California; ${ }^{3}$ Department of Plastic Surgery, University Hospitals Cleveland, Case Western Reserve University, Cleveland, Ohio.

Address for correspondence and reprint requests: Christopher J. Salgado, M.D., Department of Plastic Surgery, University Hospitals osteomyelitis, several animal models have been created for in vivo investigation of osteomyelitis.

Initially, studies by Rodet, and later by Lexer, simply injected attenuated staphylococci into rabbits and determined that acute progressive medullary infection was impossible by intravenous injection of staphylococci alone. Subsequent investigators attempted to inject staphylococci intravenously and directly onto the bone. Although they were able to successfully produce osteomyelitic lesions, few infections were progressive and rarely simulated human disease. ${ }^{5}$

In 1941, Scheman et al created a reproducible model of chronic osteomyelitis in rabbits by injecting sodium morrhuate and staphylococci directly into the tibial metaphysis of rabbits. They concluded that sodium morrhuate created aseptic necrosis of bone, providing

Cleveland, OH 44106 (e-mail: salgado_plastics@hotmail.com).

Osteomyelitis; Guest Editors, Christopher J. Salgado, M.D., and Lawrence B. Colen, M.D.

Semin Plast Surg 2009;23:148-154. Copyright (C) 2009 by Thieme Medical Publishers, Inc., 333 Seventh Avenue, New York, NY 10001, USA. Tel: $+1(212)$ 584-4662.

DOI 10.1055/s-0029-1214167. ISSN 1535-2188. 
the soil necessary for progressive infection leading to osteomyelitis. $^{6}$

Based on these initial studies, researchers were able to create models using a variety of animals to investigate different disease types and stages. Smaller animals allow easy handling and are cost-effective but are limited by their inability to survive therapeutic antibiotic doses and multiple procedures. Larger animals are more tolerant to therapeutic antibiotic doses and multiple procedures but are limited by their availability, increased costs, and potentially aggressive nature. ${ }^{7}$

Therefore, a single animal model that accommodates funding and resources and allows evaluation of treatment, pathogenic organisms, disease stage, and classification does not exist.

What follows is a literature review of the animal models created to study osteomyelitis. The models were chosen based on historical relevance and clinical applicability.

\section{METHODS}

A PubMed and OVID search was performed on March 31, 2008, using keywords osteomyelitis, animal model (rabbit, rat, mouse, avian, dog, sheep, and goat), and experimental osteomyelitis. The objective of this review was to provide a literature review of the animal models created to study osteomyelitis. The models were chosen based on historical relevance and clinical applicability.

\section{LITERATURE REVIEW}

\section{Rabbit Models}

The first attempt to create an animal model to study osteomyelitis was performed by Rodet in 1884. Rodet planned to induce osteomyelitis in rabbits using intravenous injections of Staphylococcus aureus. His attempts were highly unsuccessful, and he established that intravenous injection of bacteria would result in high mortality and low yields of osteomyelitis. ${ }^{8}$

After several attempts at creating a model for osteomyelitis, Scheman et al reported the first reproducible model with chronic, progressive osteomyelitis in rabbits. Histologic evaluation of tibias from a group receiving 5\% sodium morrhuate and a $S$. aureus suspension revealed suppuration in the bone and soft tissues, new bone formation, subperiosteal abscess, and destruction of the epiphysis and epiphyseal growth plate. The success of the model of Scheman et al was attributed to use of sodium morrhuate, a sclerosing agent that produces aseptic necrosis of bone. ${ }^{6}$

In 1970, Norden and Kennedy enhanced the model of Scheman et al by studying bacterial inoculation with and without morrhuate, thereby establishing the requirement of both agents in the formation of osteo- myelitis. Animals injected with either sodium morrhuate or Staphylococcus alone did not have pathologic evidence or cultures suggestive of osteomyelitis at 14 or 60 days. In contrast, all animals that received morrhuate and the bacterial suspension initially yielded organisms from inoculated tibia. Ninety percent of these infected tibias yielded organisms when the animals were sacrificed at 60 and 180 days. Radiographs revealed abscesses, disruption of periosteum, distortion of normal bone architecture, and new bone formation. ${ }^{9}$

Following the successes of Norden and Kennedy, Andriole et al created a model that would maintain chronic staphylococcal osteomyelitis for a period of 1.5 years. Experimental animals received an intramedullary injection of Giorgio staphylococci and insertion of a stainless steel pin. Some animals received a simple fracture before the pin insertion. Control groups did not receive the bacterial inoculation. Animals with bacterial inoculation but without intramedullary pin placement did not produce evidence of osteomyelitis by tissue examination, radiographs, or tissue culture. Eighty-eight percent of rabbits with both bacterial inoculation and pinning produced clinical, radiographic, and culture evidence of chronic osteomyelitis. ${ }^{10}$

Early rabbit models of osteomyelitis established that a foreign substance or trauma was necessary to create a chronic, progressive infection. These models rely on the virulence of Staphylococcus to create an animal form of the human disease.

Unlike previous investigators, Johansson et al were able to create a model with anaerobic organisms. Animals received sponges soaked with $1 \mathrm{~mL}$ lactated Ringer solution in their left femurs and $1 \mathrm{~mL}$ of $10^{7}$ colony forming units (cfu) of Bacteroides fragilis in their right femurs after the treatment of the proximal metaphysis with $5 \%$ sodium morrhuate. No animal had fulminant sepsis during the study. All animals had a bilateral focal increase of radionuclide activity in the proximal tibia at 5 weeks, though it was higher on the inoculated side. All but one of the inoculated sides yielded $B$. fragilis on anaerobic culture. On inspection, all cancellous bone surrounding the implanted sponge was sclerotic, and several microabscesses were found. Histologic specimens demonstrated osteomyelitis bilaterally, suggesting the development of both contiguous and hematogenous osteomyelitis. ${ }^{11}$

As it was established that osteomyelitis is a disease that is reproducible in rabbit models, investigators have been searching for models to improve therapy. Schulz and colleagues created a rabbit model with the aim to perform more local therapeutic strategies on the infected bone, thus avoiding the toxicities associated with systemic therapy. A biopsy needle was inserted into the intramedullary cavity of the proximal femur, from which $1 \mathrm{~mL}$ of bone marrow was extracted and replaced with $0.1 \mathrm{~mL}$ $5 \%$ sodium morrhuate and $0.1 \mathrm{~mL}$ of a Staphylococcus 
suspension (Kanin strain, $3 \times 10^{6} \mathrm{cfu} / \mathrm{mL}$ ). The needle was flushed and left in situ. One hundred percent of the animals developed osteomyelitis based on clinical, histologic, and radiographic findings. Schulz et al explained that these findings were the result of employing two techniques to create a bone marrow lesion: bone marrow extraction resulting in destruction of local medullary blood vessels from systemic circulation; and use of a sclerosing agent in combination with a foreign body (leaving the pin in situ). ${ }^{12}$

Rabbit models created to study osteomyelitis show the importance of preparing the bone prior to inoculation for chronic, progressive infection to develop. Now that a rabbit model exists, several investigators, like Schulz et al, are using these resources to develop methods of applying local therapies to avoid complications of systemic antibiotics and to improve efficacy.

\section{Rat Models}

Though not initially used to study osteomyelitis, rats have become a popular animal model following the work done by Zak et al, which was later expanded by Rissing et al. In these studies, rats received an injection of 5\% sodium morrhuate followed by a $S$. aureus suspension into the tibial metaphysis. This model was able to establish a chronic infection, yielding a large and stable number of organisms for an extended period of time. ${ }^{13,14}$

Previously, the animal models described relied on a bacterial suspension in combination with sclerosing agents or foreign bodies to induce osteomyelitis. Spagnolo et al avoided alterations in the medullary circulation caused by sclerosing agents and the formation of a glycocalyx by $S$. aureus adhering to foreign bodies by using fibrin glue. The anterior tibial metaphysis were inoculated with fibrin glue and $S$. aureus or fibrin glue alone. Animals receiving bacteria with fibrin glue developed osteomyelitis in $90 \%$ of cases and were similar to human disease based on radiographic, pathologic, and histologic examination. ${ }^{15}$

A complication encountered with treating osteomyelitis associated with biomaterial implantation is the formation of a biofilm. Biofilms make treatment difficult secondary to the development of resistance to antibiotics. Gracia et al developed a model to study the effects of antibiotics against bacteria within biofilms. Stainless steel rods were coated with biofilm bacteria ( $S$. aureus strain ATCC 29213 slime-producing variety) and implanted into rats. The rats also received a bacterial suspension at the surgery site. The animals were subsequently treated with tobramycin, vancomycin, or cefuroxime. Treatment with cefuroxime was shown to inhibit the colonization of bone and reduce but not eliminate bacteria from the implant. Treatment with tobramycin was not effective. Vancomycin was not able to eradicate the biofilm but was partially effective against infection. ${ }^{16}$
Biofilms were also formed in vitro and subjected to antibiotics. The number of viable adherent bacteria was determined using ATP-bioluminescence. A comparative study on the efficacy of the three antibiotics showed that both systems were closely related. This finding offers the possibility of using an in vitro assay for background information before conducting an animal experiment. ${ }^{16}$

A follow-up study by the same group created a rat model using precolonized implants in the absence of a bacterial suspension at the site of surgery. All animals demonstrated evidence of osteomyelitis at the end of the experimental period, showing that the bacterial suspension used by Garcia et al is not necessary. Again, this study confirmed the effectiveness of cefuroxime in eradicating biofilm bacteria. ${ }^{17}$

Previous models used to create osteomyelitis relied on bacterial inoculation and additional tissue manipulation to induce infection, including use of sclerosing agents, incorporation of foreign bodies, fibrin glue, and sealants to prevent bacterial leakage. Lucke et al created a rat model to mimic intramedullary nailing. To provide a model that approximates human disease, further promoters of infection were not used. It was found that acute destructive osteomyelitis was created with as little as $10^{2} \mathrm{cfu} / 10 \mu \mathrm{L}$. Also, the use of sterile solid K-wires simulated a clinical situation in which biofilm formation occurs in vivo after foreign body implantation. ${ }^{18}$

Based on the models established, several investigators have evaluated the efficacy of various antibiotics and new carrier systems for local therapy. Rats are favored as animal models due to their small size, low cost, and established models of developing osteomyelitis. Though effective, rat models are also limited by their small size, inability to tolerate multiple interventions, and the difficulty of evaluating complex surgical techniques. ${ }^{15}$

\section{Mouse Models}

Mice are popular laboratory animals used to study a variety of diseases and pathologic processes. Their popularity in research has resulted in superior knowledge of the function and regulation of the immune system. On the basis of established information, a mouse model was developed to study osteomyelitis and the resulting immune response to the infectious process.

Using a previously created rabbit model, Chadha et al developed a mouse model to apply knowledge about the immune system to study its response to osteomyelitis $(\mathrm{OM})$. Twenty-seven mice were divided into two groups: seven control mice in which a fracture of the right proximal tibial growth plate was created, and 20 mice that received an intravenous injection of $S$. aureus (strain LS-1) along the fracture. 
Seven days after the intervention, the right and left tibias were harvested along with the spleen, lymph nodes, and peripheral blood. The tibias underwent histologic, microbiological, and molecular analysis. Lymph nodes harvested underwent flow cytometric analysis.

The nontraumatized legs of the experimental animals appeared normal with no evidence of infection. Although the overlying skin of the traumatized leg appeared normal, there was evidence of abscess formation in the proximal tibia of all samples. Histologic examination of the traumatized limbs revealed distortion at the hypertrophic zone of the physis, and damage to the zones of vascular invasion and mineralization was observed. S. aureus was recovered from all samples of the traumatized limbs of the experimental group, but none was isolated from the contralateral, nonfractured limb. All samples from the control group remained sterile.

Unlike other models described, molecular evaluation for the presence of infection was done using DNA extracted from the tibia and was subjected to polymerase chain reaction with use of broad-range eubacterial primers. An electrophoresis gel revealed only positive bands from infected traumatized limbs, corresponding with the 834-bp sequence product of the bacterial $16 \mathrm{~S}$ ribosomal RNA gene. The nontraumatized limb of experimental animals and all limbs from control animals had no banding.

The model created by Harbinder et al demonstrates the ability to use the bank of existing information on the mouse immune system and the ability to create a reproducible model of acute hematogenous osteomyelitis in mice. Additionally, by using molecular analysis, an alternative method for detecting infection with $S$. aureus is possible without using the microbiology laboratory. ${ }^{19}$

\section{Avian/Chick Models}

Animal models initially used to study osteomyelitis relied on bone trauma, either chemical or mechanical, to induce infection. These models, though beneficial to study posttraumatic osteomyelitis as seen in adults, fail to provide insight on the more commonly occurring acute hematogenous osteomyelitis seen in children. Emslie and Nade created a model using chickens to help study acute hematogenous osteomyelitis. Thirtyeight chickens were used to study the natural history of acute hematogenous osteomyelitis during the first 8 days after injection of $S$. aureus (coagulase positive, phage $6 / 42 \mathrm{E} / 53 / 77 / 83 \mathrm{~A} / 84$ ) into a wing vein. Osteomyelitis developed in 27 of the 28 chickens that were sacrificed 24 hours or more after inoculation. Histologic examination of the experimental abscess demonstrated that bacteria were deposited initially within a metaphyseal vessel, a branch of the nutrient artery terminating in the zone of cartilage transformation in the growth plate.
These findings supported earlier studies that sluggish blood flow predisposed to bacterial disposition. The presence of endothelial gaps in the tips of growing metaphyseal vessels also plays a critical role in the initiation of staphylococcal osteomyelitis.

Another finding was the presence of vascular tunnels that pass through the growth plate to the epiphyseal cartilage, suggesting that bacterial translocation into these vessels allows access into the epiphysis. The obliteration of these vessels once a complete subchondral bone plate forms over the growth plate helps explain the relationship between clinical osteomyelitic syndromes and the vascular patterns found in different age groups.

The model established by Emslie and Nade is important because it provides a basis to study the natural histologic history of the development of acute hematogenous osteomyelitis. This model provides a method of studying osteomyelitis without the presence of associated trauma; it is also inexpensive and easily reproducible. ${ }^{20}$

\section{Canine Models}

Despite the advances in antibiotics and systemic therapy, the success of treating chronic osteomyelitis and implant infection remained inconsistent. Small animal models had been developed and were effective in evaluating osteomyelitis but were unable to accommodate clinically relevant implant devices and tolerate multiple procedures. Deysine et al developed a model of infecting the tibia with an injection of staphylococci into the nutrient tibial artery. ${ }^{21}$ This model created a disease that was believed to be different from human osteomyelitis and did not allow for the evaluation of infection secondary to foreign body placement.

Based on these needs, Fitzgerald created a canine model for chronic osteomyelitis to evaluate the role of surgical procedures, foreign body reactions, and the effectiveness of local antimicrobial therapy. The intramedullary space of the proximal tibia was infected with $S$. aureus and filled with polymethylmethacrylate (PMMA) cement. Experimental animals received immediate or delayed gentamicin-impregnated PMMA cement after bacterial inoculation. PMMA cement after bacterial inoculation resulted in osteomyelitis in 100\% of the tibias. Gentamicin-impregnated PMMA cement was effective only when used prophylactically and not for treatment. This was suggested by the failure of gentamicin-impregnated PMMA cement to eradicate organisms in tibias with established infection. ${ }^{22}$

After the work by Fitzgerald, a model was created by Petty et al to evaluate and compare the incidence of infection associated with commonly used materials in orthopedic surgery. The medullary canal of the proximal femur was inoculated with $S$. aureus, 
Staphylococcus epidermidis, or Escherichia coli and was followed by the insertion of a foreign body cylinder (stainless steel alloy, chromium-cobalt alloy, high-density polyethylene, or PMMA). It was found that insertion of any foreign material significantly increased the risk of infection with $S$. aureus, although only PMMA increased the risk of infection when less than $1000 \mathrm{cfu}$ of $S$. aureus were used. Again, only PMMA increased the risk of infection in femurs infected with only S. epidermidis or E. coli. ${ }^{23}$

The effectiveness of local therapy established by previous models has led to the investigation of safer, biodegradable carriers for local antimicrobial therapy. Garvin and colleagues investigated the effectiveness of a polylactide/polyglycolide copolymer carrier for local therapy. Infection was established using the model created by Fitzgerald. Four weeks after establishing infection, the wounds were debrided, and therapy was initiated. Animals were treated with parenteral antibiotics, a gentamicin-impregnated PMMA implant, or a gentamicin-impregnated polylactide/polyglycolide copolymer. Six weeks after debridement and initiation of therapy, the animals were sacrificed, tissue samples were obtained, and the implants were removed. The PMMA implants were removed intact, but only fragments of the polylactide/polyglycolide carriers could be removed because the carriers were largely reabsorbed. The animals receiving local therapy had significantly better eradication of infection than did animals treated systemically. A benefit of a biodegradeable implant is suggested by its reabsorption, eliminating the need to remove the implant after therapy. ${ }^{24}$

Use of canine models for studying osteomyelitis has led to the development and evaluation of local antimicrobial therapy. The ability of dogs to tolerate multiple procedures allows investigators to study the impact of foreign body implants as a source for infection and as a method of treatment.

\section{Sheep Models}

Most of the initial models created to study osteomyelitis involve small animals like rabbits and rats. Small animal models are limited by their inability to use routine operative techniques and tools. Therefore, a new large animal model using sheep was created to study osteomyelitis. Although dogs are available as a large animal model, their daily maintenance costs inhibits their use to study chronic osteomyelitis, which requires that followup be continued for a minimum of 6 weeks.

Kaarsemaker et al developed a large animal model using sheep to study chronic osteomyelitis. Into a small drill hole in the femur, $1 \mathrm{~mL} 3 \%$ tetradecylsodiumsulfate solution was injected, and 5 minutes later experimental animals were injected with $S$. aureus (PS 8368, phage type I) soaked in gelatin sponge strips. A sham operation was performed on the contralateral leg replacing bacteria with normal saline. One hour after the intervention, animals received a single dose of prophylactic antibiotics to prevent fatal sepsis.

Radiographs showed periosteal reaction of varying degrees, cortical and trabecular lysis, cortical thickening, mild to extensive new bone formation, and sequestration. Bacterial cultures were positive for the original S. aureus strain from all but one intramedullary sample. Histologic analysis showed chronic active inflammation, abundant osteoclast activity indicating osteolysis, and woven bone formation in all cases.

The experiment by Kaarsemaker et al demonstrated a large animal model that consistently formed a chronic, progressive, and sustainable infection. Unlike previous models using implantation of an infected foreign body, this model uses a sclerosing agent that allows researchers to investigate factors influencing surgical therapy or drug delivery systems. ${ }^{25}$

\section{Goat Models}

Previous models using dogs and sheep are effective and reproducible; however, they are limited by the lack of availability and by costs of purchasing, housing, and caring for the animals. Based on these needs, Salgado et al created a model using goats to study chronic osteomyelitis. In 28 goats, a 3-mm drill hole was made and injected with $1 \mathrm{~mL} \mathrm{5 \%}$ sodium morrhuate, followed by an injection 10 minutes later with $S$. aureus. All animals received a single dose of cefazolin 1 hour postoperatively to prevent fatal sepsis. The goats were followed clinically and radiographically for the next 12 to 16 weeks and were then sacrificed.

Twenty-seven of 28 animals had histologic and radiographic evidence of osteomyelitis with no animals developing fatal sepsis (Fig. 1). Eleven animals developed wounds with drainage from the surgical site. S. aureus was isolated from the tissue of 23 of 28 animals.

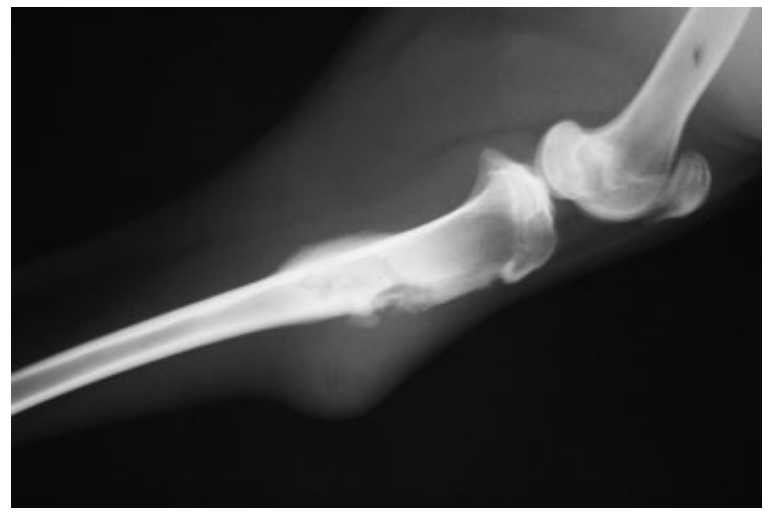

Figure 1 Lateral radiograph of goat tibia 12 weeks after inoculation with Staphylococcus aureus. Note the degree of soft tissue swelling, lucency, and periosteal reaction in the proximal tibia. 
Twenty-seven goats, including the five goats without positive cultures, did have histologic evidence of acute and chronic osteomyelitis.

Like other models, the goat model is reproducible and effective in studying chronic osteomyelitis. The goat is large enough to simulate the clinical, radiographic, and histologic characteristics of human osteomyelitis, and it allows for the evaluation and comparison of various treatment modalities for this disease. In addition, unlike other large animal models, the goat is inexpensive, is readily available, and has a temperament conducive to safe research. ${ }^{4}$

\section{Animal Models of Diagnostic Techniques}

Historically, the original model by Norden and Kennedy was modified to study diagnostic imaging techniques. Rissing outlined the use of gallium-67 $\left({ }^{67} \mathrm{Ga}\right)-$, technetium-99 $\left({ }^{99} \mathrm{Tc}\right)-$, and indium-111 $\left({ }^{111} \mathrm{In}\right)$-labeled white blood cells scintigraphy in diagnosis of acute osteomyelitis. ${ }^{5,6}$ Since his review article, new modalities have been developed and evaluated in the diagnosis of experimental osteomyelitis.

Originally, Rinsky et al compared ${ }^{99} \mathrm{Tc}$ bone scanning to conventional $\mathrm{x}$-ray techniques in the diagnosis of acute osteomyelitis in a rabbit model. His study found that standard radiography was more sensitive than ${ }^{99} \mathrm{Tc}$ scintigraphy and that there was no clear correlation between histologic diagnosis of osteomyelitis and bone scan results. ${ }^{26}$

Recently, new studies were published evaluating the usefulness of ${ }^{99} \mathrm{Tc}$ scintigraphy. In 2000, Gratz et al found that ${ }^{99} \mathrm{Tc}$-methylene diphosphonate (MDP) was sensitive but lacked specificity in the diagnosis of acute osteomyelitis. In previous studies, it was found that ${ }^{99} \mathrm{Tc}-\mathrm{MDP}$ bone scan was able to pick up disintegrated polymorphonuclear (PMN) cells in osteomyelitic bone. In 2001, Gratz et al evaluated ${ }^{99} \mathrm{Tc}$-interleukin-8 (IL-8) bone scanning in a rabbit model. Theoretically, IL-8 should be more sensitive in picking up neutrophil infiltrates. Their study found that radiography was unreliable in establishing new bone infection, whereas ${ }^{99} \mathrm{Tc}-\mathrm{IL}-8$ scan accurately identified acute osteomyelitis within 4 hours of injection. $^{27,28}$

Dams et al evaluated the performance of two new radiopharmaceuticals ${ }^{99} \mathrm{Tc}$-polyetheleneglycol (PEG) liposomes and ${ }^{99} \mathrm{Tc}$-hydrazinonicotin (HYNIC) immunoglobulin $\mathrm{G}(\mathrm{lg} G)$ in the diagnosis of chronic osteomyelitis in a rabbit model. His group concluded that the two new methods were as accurate as ${ }^{111} \mathrm{In}$ and Ga-citrate in localization of osteomyelitis. ${ }^{29}$ In multiple studies, magnetic resonance imaging (MRI) was proved to be ineffective in the diagnosis of acute osteomyelitis. Although it provides excellent anatomic detail, it has a limited role with metallic implants and does not distin- guish well between acute inflammation and tissue trauma due to recent surgery. ${ }^{30}$

Early clinical experience with positron emission tomography (PET) showed that it may be useful in diagnosis of osteomyelitis. Koort et al developed a localized osteomyelitis model in rabbit tibia by modifying a previously reported canine model to study fluorine18 fluorodeoxyglucose $\left({ }^{18} \mathrm{~F}-\mathrm{FDG}\right) \mathrm{PET}$. FDG is intensely consumed by PMN cells, and in previous animal studies its uptake was increased during acute infection. This study found that in the control animals (sham surgery), uncomplicated bone healing was associated with a temporary increase in ${ }^{18} \mathrm{~F}$-FDG uptake at 3 weeks, but it returned to almost normal by the 6th week. In the experimental animals, localized osteomyelitis resulted in an intense continuous uptake of ${ }^{18}$ F-FDG, which was higher than that of healing and intact bones at 3 weeks. ${ }^{31}$ Most recently, multiple studies have been published comparing PET scan to already existing modalities. ${ }^{32,33}$ Generally, results suggest that PET is useful in distinguishing normal from infected tissue in acute osteomyelitis, but further experiments are warranted.

\section{CONCLUSION}

Although numerous animal models exist to study both acute and chronic osteomyelitis, the search for the ideal model still persists. Evaluating chronic disease requires the ability to perform multiple procedures, to withstand current and investigative medical and surgical therapies, and yet remain inexpensive and convenient to carry out for extended periods of time. Since the earliest work by Rodet, several new models have been established and currently facilitate the evaluation of therapies and diagnostic modalities. In addition, unlike earlier studies, current studies closely simulate human disease and therapeutic modalities. Although numerous advances have been made, no single model is ideal to study all aspects of osteomyelitis, and further investigation is warranted.

\section{REFERENCES}

1. Maynor ML, Moon RE, Camporesi EM. Chronic osteomyelitis of the tibia: treatment of hyperbaric oxygen and autogenous microsurgical muscle transplantation. J South Orthop Assoc 1998;7:43-57

2. Waldvogel FA, Medoff G, Swartz MN. Osteomyelitis: a review of clinical features, therapeutic considerations, and unusual aspects (part 1-3). N Engl J Med 1970;282:198-206

3. Cierny G III, Mader JT, Penninck JJ. A clinical staging system for adult osteomyelitis. Clin Orthop Relat Res 2003; 414:7-24

4. Salgado CJ, Jamali AA, Mardini S. A model for chronic osteomyelitis using Staphylococcus aureus in goats. Clin Orthop Relat Res 2005;436:246-250 
5. Rissing JP. Animal models of osteomyelitis: knowledge, hypothesis, and speculation. Infect Dis Clin North Am 1990;4:377-390

6. Scheman L, Janota M, Lewin P. The production of experimental osteomyelitis: preliminary report. JAMA 1941; 177:1525-1529

7. Leader P, Padgett P. Animal models in biomedical research. In: Fox JC, Cohen BJ, Loew FM, eds. Laboratory Animal Medicine. San Diego, CA: Academic Press Inc; 1984: 134-139

8. Rodet A. Etude experimentale sur l'osteomyelite infectieuse. Compt Rend Acad Sci 1884;99:569-571

9. Norden CW, Kennedy E. Experimental osteomyelitis. I: a description of the model. J Infect Dis 1970;122:410-418

10. Andriole VT, Nagel DA, Southwick WO. A paradigm for human chronic osteomyelitis. J Bone Joint Surg Am 1973;55: 1511-1515

11. Johansson A, Svensson O, Blomgren G. Anaerobic osteomyelitis: a new experimental rabbit model. Clin Orthop Relat Res 1991;265:297-301

12. Schulz S, Steinhart H, Mutters R. Chronic osteomyelitis in a new rabbit model. J Invest Surg 2001;14:121-131

13. Zak O, Zak F, Rich R. Experimental staphylococcal osteomyelitits in rats: therapy with rifampin and cloxacillin alone or in combination. In: Perti P, Grassi G, eds. Current Chemotherapy and Immunotherapy. Washington, DC: American Society for Microbiology; 1982:973

14. Rissing JP, Buxton TB, Weinstein BS. Model of experimental chronic osteomyelitis in rats. Infect Immun 1985;47: 581-586

15. Spagnolo N, Greco F, Rossi A. Chronic staphylococcal osteomyelitis: a new experimental rat model. Infect Immun 1993;61:5225-5230

16. Gracia E, Laclériga A, Monzón M. Application of a rat osteomyelitis model to compare in vivo and in vitro the antibiotic efficacy against bacteria with high capacity to form biofilms. J Surg Res 1998;79:146-153

17. Monzón M, Garcia-Alvarez F, Laclériga A. A simple infection model using pre-colonized implants to reproduce chronic Staphylococcus aureus osteomyelitis and study antibiotic treatment. J Orthop Res 2001;19:820-826

18. Lucke M, Schmidmaier G, Sadoni S. A new model of implant-related osteomyelitis in rats. J Biomed Mater Res B Appl Biomater 2003;67:593-602

19. Chadha HS, Fitzgerald RH, Wiater P, et al. Experimental acute hematogenous osteomyelitis in mice. I. Histopathological and immunological findings. J Orthop Res 1999;17: 376-381
20. Emslie KR, Nade S. Acute hematogenous staphylococcal osteomyelitits. A description of the natural history in an avian model. Am J Pathol 1983;110:333-345

21. Deysine M, Rosario E, Isenberg HD. Acute hematogenous osteomyelitis: an experimental model. Surgery 1976;79: 97-99

22. Fitzgerald RH. Experimental osteomyelitis: description of a canine model and the role of depot administration of antibiotics in the prevention and treatment of sepsis. J Bone Joint Surg Am 1983;65:371-380

23. Petty W, Spanier S, Shuster JJ. The influence of skeletal implants on incidence of infection. Experiments in a canine model. J Bone Joint Surg Am 1985;67:1236-1244

24. Garvin KL, Miyano JA, Robinson D. Polylactide/polyglycolide antibiotic implants in the treatment of osteomyelitis. A canine model. J Bone Joint Surg Am 1994;76:15001506

25. Kaarsemaker S, Walenkamp GHIM, Bogaard AEJ. New model for chronic osteomyelitis with Staphylococcus aureus in sheep. Clin Orthop Relat Res 1997;339:246-252

26. Rinsky L, Goris ML, Schurman DJ, Nagel DA. Technetium-99 bone scanning in experimental osteomyelitis. Clin Orthop Relat Res 1977;128:361-366

27. Gratz S, Doerner J, Oestmann JW. 67Ga-citrate and 99T $\mathrm{cm}-\mathrm{MDP}$ for estimating the severity of vertebral osteomyelitis. Nucl Med Commun 2000;21:111-120

28. Gratz S, Rennen HJ, Boerman OC, et al. Technetium-99interleukin-8 for imaging acute osteomyelitits. J Nucl Med 2001;42:1257-1264

29. Dams ETHM, Nijhof MW, Boerman OC. Scintigraphic evaluation of experimental chronic osteomyelitis. J Nucl Med 2000;41:896-902

30. Ledermann HP, Kaim A, Bongartz G. Pitfalls and limitations of magnetic resonance imaging in chronic posttraumatic OM. Eur Radiol 2000;10:1815-1823

31. Koort JK, Makinen TJ, Knuuti J. Comparative ${ }^{18}$ F-FDG PET of experimental Staphylococcus aureus osteomyelitis and normal bone healing. J Nucl Med 2004;45:14061411

32. Jones-Jackson L, Walker R, Purnell G. Early detection of bone infection and differentiation from post-surgical inflammation using 2-deoxy-2-[ $\left[{ }^{18} \mathrm{~F}\right]$-fluoro-d-glucose positron emission tomography (FDG-PET) in an animal model. J Orthop Res 2005;23:1484-1489

33. Makinen TJ, Lankinen $\mathrm{P}$, Poyhonen T. Comparison of ${ }^{18} \mathrm{~F}$-FDG and ${ }^{68} \mathrm{Ga}$ PET imaging in the assessment of experimental osteomyelitis due to Staphylococcus aureus. Eur J Nucl Med Mol Imaging 2005;32:1259-1268 